\title{
Quantificação do controle químico de plantas daninhas através de diferentes formas de absorção
}

\author{
Chemical control quantification of weeds through different uptake forms
}

\author{
Cleber Daniel de Goes Maciel ${ }^{*}$; Juliana Parisotto Poletine ${ }^{1}$; Jamil Constantin²; \\ Celso Eduardo Jardim ${ }^{3}$; Rodrigo dos Santos Bernardo ${ }^{3}$
}

\begin{abstract}
Resumo - O trabalho objetivou quantificar a intensidade de ação de herbicidas no controle de Brachiaria decumbens (BRADC) e Digitaria horizontalis (DIGHO), através de diferentes formas de absorção. Para tanto, três experimentos foram conduzidos em estufas plásticas representados pelos herbicidas atrazine (3,00 kg i.a. ha ${ }^{-1}+$ óleo mineral Assist $\left.{ }^{\circledR} 0,5 \% \mathrm{v} / \mathrm{v}\right)$, oxyfluorfen $(0,72 \mathrm{~kg}$ i.a. ha $\left.{ }^{-1}\right)$ e isoxaflutole $\left(0,06 \mathrm{~kg}\right.$ i.a. $\left.\mathrm{ha}^{-1}\right)$, aplicados em pós-emergência inicial. Três possibilidades de absorção (radicular, foliar e radicular + foliar) e uma testemunha foram estudadas em solos de textura arenosa e argilosa, constituindo fatorial 4 x 2, com cinco repetições. Para o isolamento da absorção dos herbicidas foi utilizado canudos plásticos e papel alumínio para garantir a presença ou ausência de proteção das plântulas ou solo. Observou-se que a absorção foliar e radicular de isoxaflutole controlou eficientemente a BRADC nos solos de textura arenosa e argilosa aos 15 DAA (dias após aplicação). Para DIGHO, o isoxaflutole proporcionou controle eficiente através da via radicular, na ordem de 1,9 e 5,6 vezes, superior a via foliar para os solos arenoso e argiloso aos 15 DAA. A absorção foliar de oxyfluorfen controlou a BRADC (>93,0\%) na ordem de 16,8 e 15,1 vezes superiores a via radicular para os solos de textura arenosa e argilosa. A espécie DIGHO não foi satisfatoriamente controlada pelos herbicidas atrazine e oxyfluorfen por nenhuma das diferentes vias de absorção e tipos de solo.

Palavras-chave: Herbicida, metodologia, Brachiaria decumbens, Digitaria horizontalis
\end{abstract}

Abstract - The work aimed to quantify the intensity of herbicides action in Brachiaria decumbens (BRADC) and Digitaria horizontalis (DIGHO) control, through different uptake forms. This way, three experiments were carried out in plastic greenhouse conditions represented by atrazine $\left(3.00 \mathrm{~kg} \mathrm{ha}^{-1}+\right.$ mineral oil $\left.0.5 \% \mathrm{v} \mathrm{v}^{-1}\right)$, oxyfluorfen $\left(0.72 \mathrm{~kg} \mathrm{ha}^{-1}\right)$ and isoxaflutole $\left(0.06 \mathrm{~kg} \mathrm{ha}^{-1}\right)$ herbicides, applied in initial post-emergence. Three uptake possibilities (root, foliar and root + foliar) and a check treatment were studied, in sandy and clay soils, constituted by $4 \times 2$ factorial design, with five replications. For the isolation of uptake herbicides it was used plastic tubes and aluminum paper to guarantee the presence or absence of seedlings or soil protection. It was observed that isoxaflutole foliar and root uptake controlled BRADC efficiently in sandy and clay soils to 15 DAA (days after application). For DIGHO, isoxaflutole provided efficient control by root route, in the order of 1.9 and 5.6 times, superior than foliar route for sandy and clay soils to 15 DAA. Oxyfluorfen foliar uptake controlled BRADC (>93\%) in the order of 16.8 and 15.1

\footnotetext{
${ }^{1}$ Prof. Dr., Depto de Ciências Biológicas e Fitossanitárias, Escola Superior de Agronomia de Paraguaçu Paulista/SP - FUNGE/ESAPP. C.P. 88, 19700-000. Paraguaçu Paulista, SP. *e-mail: macielconsultoria@ hotmail.com

${ }^{2}$ Prof. Adjunto Dr., Depto de Agronomia Universidade Estadual de Maringá - UEM, 87020-900. Maringá, PR

${ }^{3}$ Alunos do Curso de Agronomia, Escola Superior de Agronomia de Paraguaçu Paulista/SP - FUNGE/ESAPP
}

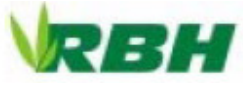


times superior than root route for sandy and clay soils. DIGHO specie was not satisfactorily controlled by atrazine and oxyfluorfen herbicides for none of different uptake routes and soil types.

Key-words: Herbicide, methodology, Brachiaria decumbens, Digitaria horizontalis.

\section{Introdução}

A utilização de herbicidas na agricultura vem sendo consagrada como um dos principais métodos de controle exercido sobre as plantas daninhas, visto que, quando mal controladas podem causar perdas de produtividade. Segundo Karam \& Melhorança (2004), as perdas nas culturas em função da interferência imposta pelas plantas daninhas tem sido descritas na ordem de $13,1 \%$, sendo que em casos onde não tenha realizado nenhum método de controle esta redução pode chegar a aproximadamente $85,0 \%$.

Dentre as plantas daninhas consideradas problemáticas no Brasil, Brachiaria decumbens e Digitaria horizontalis merecem destaque, por exercerem competição sobre as culturas anuais e causarem prejuízos significativos à qualidade e rendimento da produção (Kissmann, 1991; Martins, 1994; Souza et al., 1996; Merotto Jr. et al., 1997; Spacer \& Vidal, 2000).

A importância de se conhecer os princípios fisiológicos das plantas relacionados à absorção e translocação de herbicidas é extremamente importante para maximização dos efeitos desses compostos, assim como para avaliação das causas de seu possível insucesso. Com a adequação da modalidade de aplicação, em função da melhor via de absorção, ampliase, a possibilidade de controle das plantas infestantes, evitando desperdícios e melhorando o direcionamento do produto, e conseqüentemente, reduz os riscos à cultura e ao meio ambiente (Vidal \& Merotto Jr., 2001).

As vias de absorção de herbicidas podem ser através de estruturas aéreas (folhas, caules, flores e frutos), subterrâneas (raiz, rizoma, estolão, tubérculo) e por sementes; sendo as folhas, entretanto, o principal órgão das plantas daninhas envolvido na penetração de herbicidas aplicados em pós-emergência (Silva et al., 2000; Procópio et al., 2003). Para alguns herbicidas utilizados em pósemergência como a atrazine são relatadas possibilidades de absorção pelas vias radicular e foliar (Santos \& Rozanski, 1979; Almeida, 1989; Rodrigues \& Almeida, 2005). No entanto, apesar das recomendações técnicas priorizarem a utilização da atrazine em préemergência, haja vista a melhor absorção radicular e controle, sua aplicação em pósemergência inicial além de também proporcionar a absorção foliar, disponibiliza parte da calda de pulverização em efeito residual, minimizando o surgimento de reinfestação ainda no período crítico de prevenção da interferência. Semelhante a atrazine, outros herbicidas também podem ser absorvidos pelas vias foliar e radicular sendo predominantemente o meristema apical, raízes e colo das plântulas sensíveis, como ocorre, por exemplo, com o isoxaflutole (Vidal, 1997; Adoryan et al., 2002; Procópio et al., 2003; Rodrigues \& Almeida, 2005).

A possibilidade de se investigar as vias de absorção de herbicidas pelas plantas, com a escolha do método mais adequado a esse fim, depende dos objetivos experimentais, da facilidade do método e do nível de instrumentalização necessário (Devine et al., 1993). Algumas técnicas avaliam a aplicação de produtos radioativos sobre as folhas e posterior quantificação da radiação remanescente na superfície foliar após lavagem das mesmas com solventes próprios (Al Khatib 
et al., 1992; Gaskin \& Holloway, 1992; Wahlers et al., 1997; Sprague et al., 1999), ou imersão de pequenos pedaços de tecidos em solução tamponada com herbicida (Lichtner, 1983; Devine et al., 1987).

Nesse contexto, o uso de métodos de baixo custo para se estudar as vias de absorção de herbicidas, além de permitir melhor compreensão do comportamento da molécula avaliada, possibilita a adequação de tecnologias de aplicação distintas para diferentes formulações e situações nas quais são utilizadas (Maciel et al., 2002).

O trabalho teve como objetivo quantificar a intensidade de ação dos herbicidas atrazine, isoxaflutole e oxyfluorfen através do controle das espécies $B$. decumbens e $D$. horizontalis, utilizando-se diferentes formas de absorção e duas condições de solo.

\section{Material e métodos}

Três experimentos foram conduzidos sob estufas plásticas (cultivo protegido) da Escola Superior de Agronomia de Paraguaçu Paulista, no Município de Paraguaçu Paulista/SP, utilizando-se delineamento experimental inteiramente casualizado com cinco repetições. Cada unidade experimental constituiu-se por um vaso plástico, contendo 2,0 $\mathrm{kg}$ de solo, no qual as espécies $B$. decumbens e $D$. horizontalis foram separadamente cultivadas. As plantas daninhas foram semeadas a $0,5 \mathrm{~cm}$ de profundidade, em duas linhas paralelas e, após a emergência, estabeleceu-se a densidade de oito plântulas por espécie. Essa densidade equivaleu a uma infestação de 380 plantas $\mathrm{m}^{-2}$, comumente verificado em condições de campo.

Os tratamentos foram dispostos em esquema fatorial $4 \mathrm{x} 2$, com quatro possibilidades ou vias de absorção de herbicidas pelas plantas daninhas e dois tipos de solos (textura arenosa e argilosa). Os solos utilizados como substratos nos experimentos foram classificados como Latossolo Vermelho distrófico, de textura arenosa, e Argissolo Vermelho distrófico, de textura argilosa, e apresentavam as seguintes proporções, respectivamente, entre os constituintes: areia $(80,3$ e $23,4 \%)$, argila $(12,2$ e $56,6 \%)$, silte $(7,5$ e 20,0\%) e matéria orgânica $\left(7,0\right.$ e 5,0 $\left.\mathrm{g} \mathrm{dm}^{-3}\right)$.

As diferentes vias de absorção dos herbicidas foram estabelecidas pela ausência ou presença de proteção da parte aérea das plântulas e/ou da superfície do solo, proporcionando exposição e/ou isolamento da planta ou do solo, respectivamente, à deposição da pulverização, conforme método desenvolvido e descrito por Maciel et al. (2002). Pela combinação de tratamentos envolvendo a proteção das plantas daninhas (canudos plásticos) ou do solo (papel alumínio), foi possível estabelecer-se quatro possibilidades de absorção dos herbicidas: a) plântulas protegidas e solo desprotegido absorção radicular (subterrânea); b) plântulas desprotegidas e solo protegido - absorção foliar (aérea); c) plântulas solo e sem proteção absorção foliar e radicular (subterrânea e aérea); d) um tratamento apresentando ausência de absorção de herbicida - testemunha sem aplicação (Figura 1). Meia hora após as aplicações dos herbicidas, os canudos plásticos e o papel alumínio foram cuidadosamente removidos, evitando-se a contaminação das vias de absorção. Aproximadamente 24 horas após a aplicação dos herbicidas, todas as unidades experimentais foram irrigadas por subsuperfície, colocando-se os vasos em bandejas com lâminas d'água de volume previamente estabelecido através de pesagem, de tal forma que diariamente o umedecimento uniforme dos solos ocorre-se por capilaridade através de furos no fundo dos vasos, mantendoos com umidade em torno de $80 \%$ da capacidade de campo.

Esse procedimento foi adotado objetivando-se evitar o contato da água com a 
parte aérea das plantas, assim como o distúrbio da superfície do solo onde os herbicidas foram depositados e, consequentemente, a ocorrência de prováveis irregularidades na movimentação dos herbicidas. Desta forma, no referido estudo, os herbicidas não tiveram o auxílio de lâminas d'água sobre a superfície do solo para posicioná-los mais facilmente no contato com as raízes ou outras partes subterrâneas, o que simula a condição de presença de umidade no solo sem ocorrência de chuvas logo após aplicação.

Os herbicidas atrazine $\left(3,00 \mathrm{~kg}\right.$ i.a. $\mathrm{ha}^{-1}$ + óleo mineral Assist $\left.{ }^{\circledR} 0,5 \% \mathrm{v} \mathrm{v}^{-1}\right)$, oxyfluorfen $\left(0,72 \mathrm{~kg}\right.$ i.a. ha $\left.{ }^{-1}\right)$ e isoxaflutole $\left(0,06 \mathrm{~kg}\right.$ i.a. $\mathrm{ha}^{-}$ ${ }^{1}$ ) foram estudados em experimentos e épocas distintas. A aplicação dos herbicidas foi realizada utilizando-se um pulverizador costal pressurizado a base de $\mathrm{CO}_{2}$, equipado com barra e pontas XR110.02-VS, aplicando-se o equivalente a $200 \mathrm{~L} \mathrm{ha}^{-1}$ de calda. No momento das pulverizações, o solo encontrava-se úmido, a temperatura, a umidade relativa do ar e velocidade dos ventos foram de $26,6^{\circ} \mathrm{C}, 72,0 \%$ e $3,4 \mathrm{~km} \mathrm{~h}^{-1} ; 23,9^{\circ} \mathrm{C}, 68 \%$ e $3,3 \mathrm{~km} \mathrm{~h}^{-1}$ e de $27,9^{\circ} \mathrm{C}, 57 \%$ e $2,7 \mathrm{~km} \mathrm{~h}^{-1}$ para os herbicidas atrazine, oxyfluorfen e isoxaflutole, respectivamente. Nessa mesma época as plantas daninhas apresentavam-se com estádio com três a quatro folhas.

A contribuição relativa de cada via de absorção (radicular, foliar, e ou ambas) para absorção total, ou a determinação da via de absorção predominante nas condições estudadas foi realizada apenas de forma indireta, por meio de avaliações visuais (notas) da porcentagem de controle, conforme proposto por Maciel et al. (2002). Portanto, aos 3; 9, e 15 dias após a aplicação dos herbicidas (DAA) para B. decumbens, e aos 3, 7 e 14 DAA para $D$. horizontalis, essa avaliação foi realizada de forma que $0 \%$ representava a ausência de danos nas espécies estudadas e $100 \%$ para o controle total ou morte das plantas daninhas (SBCPD, 1995). Considerou-se eficiente $o$ tratamento que apresentou porcentagem de controle superior a $80,0 \%$.

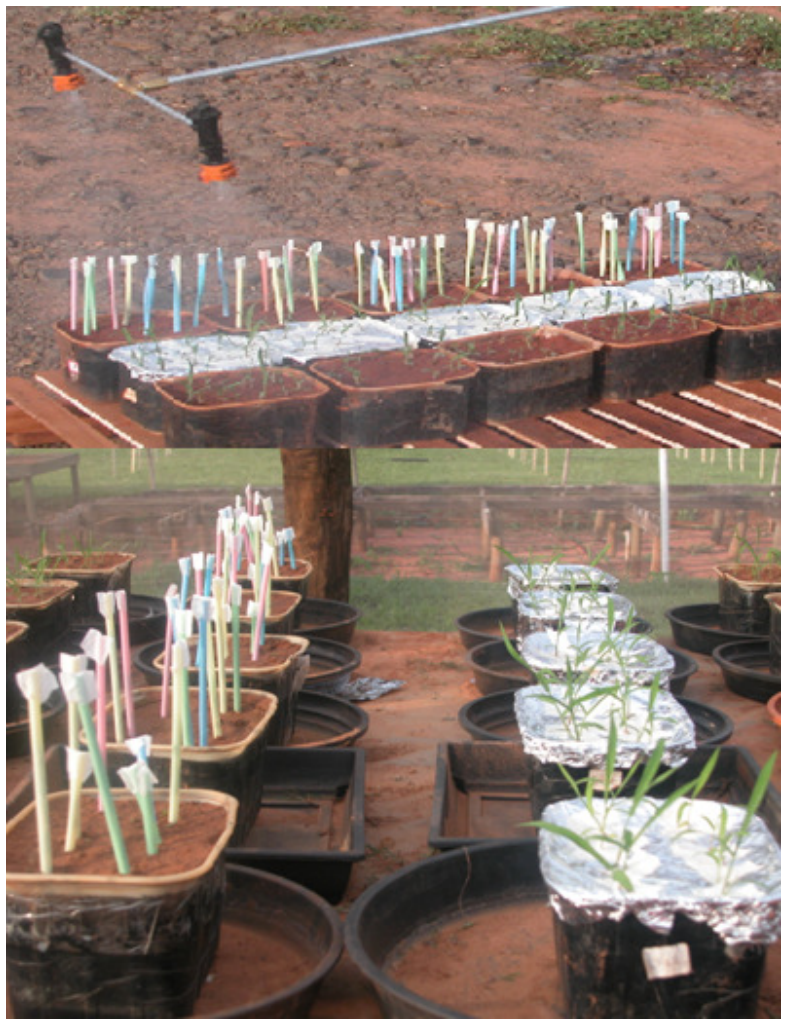

Figura 1. Representação esquemática, ilustrando a aplicação dos herbicidas atrazine, isoxaflutole e oxyfluorfen com o sistema de isolamento das plântulas ou do solo.

Ao final do período experimental, para determinação da matéria seca, as partes aéreas das plantas foram coletadas e devidamente acondicionadas em estufa de circulação forçada de ar a $65^{\circ} \mathrm{C}$, por período de três dias.

Os dados obtidos foram submetidos à análise de variância pelo teste $\mathrm{F}$ e as médias dos tratamentos comparadas entre si pelo teste $t$, a $5 \%$ de probabilidade.

\section{Resultados e discussão}

Aos 3 DAA, a absorção foliar de atrazine e oxyfluorfen proporcionou controle significativamente superior à absorção radicular para os solos de textura arenosa e 
argilosa (Tabela 1). Aos 9 DAA, iniciaram-se os efeitos positivos da associação das absorções radicular + foliar da atrazine, proporcionando controle superior aos obtidos pelas vias isoladas, sendo que aos15 DAA esse efeito tornou-se mais pronunciado. Aos 15 DAA, os níveis de controle de $B$. decumbens através da absorção foliar não foram satisfatórios $(\leq 36,0 \%)$ para ambos os solos, mas apresentaram-se superiores à absorção radicular em 9,5 e 3,9 vezes, respectivamente. Maciel et al. (2002) constataram resultados semelhantes, evidenciando melhor performance de controle de Brachiaria plantaginea pela atrazine via absorção foliar em detrimento à radicular, assim como consideraram imprescindível a necessidade de precipitação após aplicação para haver predomínio da absorção radicular.

Nesse sentido, consolida a informação, para mais uma espécie de planta daninha, de que para atrazine mesmo em solo apresentando-se com condição de umidade no momento e após aplicação, é ainda necessário que ocorra uma precipitação ou irrigação após a aplicação para incorporação e, consequentemente, absorção efetiva desse herbicida através da via radicular de plântulas já emergidas.

Para o isoxaflutole, os sintomas de injúrias na $B$. decumbens iniciaram a partir dos 9 DAA (Tabela 1), em função da baixa luminosidade provocado devido à condição de tempo nublado, durante aproximadamente 4 DAA. Esse possível comportamento, de retardar o aparecimento das injúrias nas plantas sensíveis, é característico de herbicidas inibidores da síntese de carotenóides. Para esse grupo de herbicidas, os sintomas típicos de branqueamento das plantas devem-se à fotooxidação e destruição da clorofila na presença de luz, gerando posterior aumento do estresse oxidativo e consequente destruição das membranas celulares (Devine et al., 1993;
Sprague et al., 1999; Vidal \& Merotto Jr., 2001).

Aos 15 DAA, o isoxaflutole absorvido pelas vias foliar e radicular, de forma isolada ou associada, promoveu controle altamente eficiente da $B$. decumbens $(100,0 \%)$ nos solos de textura arenosa e argilosa, não diferindo estatisticamente entre si. Segundo Pallet et al. (1998) e Young \& Hart (2000) o isoxaflutole assim como a sua forma ativa diketonitrile (DKN) apresentam mobilidade simplástica e apoplástica em plantas sensíveis, apesar do isoxaflutole ser absorvido preferencialmente através do sistema radicular.

Diferentemente do observado para atrazine e isoxaflutole, a absorção foliar do oxyfluorfen, a partir dos 9 DAA, proporcionou níveis eficientes de controle de $B$. decumbens (> 93,0\%), sendo, respectivamente, na ordem de 16,8 e 15,1 vezes superiores a via exclusivamente radicular (subterrânea) para os solos de textura arenosa e argilosa. Para o oxyfluorfen é importante ressaltar que apesar de sua baixa solubilidade em água $(<0,1 \mathrm{ppm})$ e, consequentemente, difícil lixiviação no solo, ainda apresenta-se capaz de ser absorvido por estruturas subterrâneas e pelo sistema radicular de plantas daninhas de emergência mais superficial (Vidal, 1997; Deuber, 2003; Rodrigues \& Almeida, 2005).

O clima no decorrer do experimento foi adequado ao desenvolvimento do milho e das plantas daninhas, que se encontravam em plena atividade metabólica na época da aplicação dos tratamentos. Todos os herbicidas promoveram controle eficiente (igual ou superior a $80 \%$ ) para Brachiaria plantaginea em todas as avaliações (Tabela 2).

$\mathrm{Na}$ avaliação aos 7 dias após a aplicação (DAA) dos tratamentos, o controle dos tratamentos foi menor que nas demais avaliações, nas quais o potencial de controle dos mesmos foi exteriorizado. 
Tabela 1. Controle (\%) de Brachiaria decumbens (BRADC), considerando as diferentes possibilidades de absorção de atrazine, isoxaflutole e oxyfluorfen e as características dos solos.

\begin{tabular}{|c|c|c|c|c|c|c|}
\hline \multirow{3}{*}{$\begin{array}{l}\text { Formas de } \\
\text { Absorção }\end{array}$} & \multicolumn{6}{|c|}{ Controle (\%) de BRADC através da absorção de atrazine } \\
\hline & \multicolumn{2}{|c|}{$3 \mathrm{DAA}^{-1 / 2}$} & \multicolumn{2}{|c|}{$9 \mathrm{DAA}$} & \multicolumn{2}{|c|}{$15 \mathrm{DAA}$} \\
\hline & Arenoso & Argiloso & Arenoso & Argiloso & Arenoso & Argiloso \\
\hline Radicular & $0,0 \mathrm{cA}^{/ 2}$ & $0,0 \mathrm{cA}$ & $4,8 \mathrm{cA}$ & $3,8 \mathrm{cA}$ & $3,8 \mathrm{cB}$ & $8,0 \mathrm{cA}$ \\
\hline Foliar & $39,0 \mathrm{aA}$ & $11,0 \mathrm{aB}$ & $48,0 \mathrm{bA}$ & $42,6 \mathrm{bA}$ & 36,0 bA & $31,4 \mathrm{bB}$ \\
\hline Radicular+foliar & $10,4 \mathrm{bA}$ & $6,0 \mathrm{bB}$ & $94,0 \mathrm{aA}$ & $58,0 \mathrm{aB}$ & $98,4 \mathrm{aA}$ & $90,4 \mathrm{aB}$ \\
\hline Testemunha & $0,0 \mathrm{cA}$ & $0,0 \mathrm{cA}$ & $0,0 \mathrm{cA}$ & $0,0 \mathrm{cA}$ & $0,0 \mathrm{dA}$ & $0,0 \mathrm{dA}$ \\
\hline$\overline{\text { Absorção (A) }}$ & \multicolumn{2}{|c|}{$126,45^{*}$} & \multicolumn{2}{|c|}{$437,05^{*}$} & \multicolumn{2}{|c|}{$2507,81^{*}$} \\
\hline Solo (S) & \multicolumn{2}{|c|}{$59,73^{*}$} & \multicolumn{2}{|c|}{$37,75^{*}$} & \multicolumn{2}{|c|}{$5,93 *$} \\
\hline$A \times S$ & \multicolumn{2}{|c|}{$41,04 *$} & \multicolumn{2}{|c|}{$24,54 *$} & \multicolumn{2}{|c|}{$9,54 *$} \\
\hline $\mathrm{CV} \%$ & \multicolumn{2}{|c|}{39,93} & \multicolumn{2}{|c|}{17,38} & \multicolumn{2}{|c|}{33,50} \\
\hline DMS $(5 \%)$ & \multicolumn{2}{|c|}{4,29} & \multicolumn{2}{|c|}{7,06} & \multicolumn{2}{|c|}{3,53} \\
\hline Formas de & \multicolumn{6}{|c|}{ Controle $(\%)$ de BRADC através da absorção de isoxaflutole } \\
\hline \multirow[t]{2}{*}{ Absorção } & \multicolumn{2}{|c|}{$3 \mathrm{DAA}$} & \multicolumn{2}{|c|}{$9 \mathrm{DAA}$} & \multicolumn{2}{|c|}{$15 \mathrm{DAA}$} \\
\hline & Arenoso & Argiloso & Arenoso & Argiloso & Arenoso & Argiloso \\
\hline Radicular & 0,0 & 0,0 & 13,8 a $\mathrm{A}$ & $11,4 \mathrm{~b} \mathrm{~B}$ & 100,0 & 100,0 \\
\hline Foliar & 3,0 & 0,0 & $5,4 \mathrm{c} \mathrm{B}$ & 8,0 с A & 100,0 & 100,0 \\
\hline Radicular+foliar & 0,0 & 0,0 & 11,6 b B & 14,6 a $\mathrm{A}$ & 100,0 & 100,0 \\
\hline Testemunha & 0,0 & 0,0 & $0,0 \mathrm{~d} \mathrm{~A}$ & $0,0 \mathrm{~d} A$ & 0,0 & 0,0 \\
\hline$\overline{\text { Absorção (A) }}$ & \multicolumn{2}{|c|}{ - } & \multicolumn{2}{|c|}{$159,54^{*}$} & & \\
\hline Solo $(\mathrm{S})$ & & & 2,72 & & & \\
\hline$A \times S$ & & - & 6,7 & & & . \\
\hline $\mathrm{CV} \%$ & & & 18 & & & 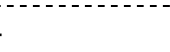 \\
\hline DMS (5\%) & & . & 1, & & & - \\
\hline Formas & Contro & e $(\%)$ de $B$ & $\mathrm{RADC}$ atra & és da absc & rção de oxy & fluorfen \\
\hline Absorção & $3 \mathrm{D}$ & $\mathrm{AA}$ & $9 \mathrm{D}$ & $\mathrm{AA}$ & $15 \mathrm{I}$ & $\mathrm{AA}$ \\
\hline & Arenoso & Argiloso & Arenoso & Argiloso & Arenoso & Argiloso \\
\hline Radicular & 0,0 c A & $3,0 \mathrm{~b} \mathrm{~A}$ & $5,8 \mathrm{~b} \mathrm{~A}$ & 6,2 c A & $9,4 \mathrm{~b} \mathrm{~B}$ & $11,8 \mathrm{~b} \mathrm{~A}$ \\
\hline Foliar & 33,0 a $A$ & 32,2 a A & 97,2 a $A$ & 93,8 a $A$ & 100,0 a $\mathrm{A}$ & 100,0 a $\mathrm{A}$ \\
\hline Radicular+foliar & 19,0 b B & 30,6 a $A$ & 97,0 a $\mathrm{A}$ & 83,0 b B & 100,0 a $\mathrm{A}$ & 100,0 a $\mathrm{A}$ \\
\hline Testemunha & 0,0 c A & $0,0 \mathrm{~b} \mathrm{~A}$ & $0,0 \mathrm{c} \mathrm{A}$ & $0,0 \mathrm{~d} A$ & 0,0 c A & $0,0 \mathrm{c} \mathrm{A}$ \\
\hline$\overline{\text { Absorção (A) }}$ & 193 & $68^{*}$ & 3189 & $26 *$ & 4398 & $2,51 *$ \\
\hline Solo $(\mathrm{S})$ & & & 21, & & 5,2 & $6^{*}$ \\
\hline$A \times S$ & & & 13 & & 5,2 & $6^{*}$ \\
\hline $\mathrm{CV} \%$ & & 40 & 6 & & & 57 \\
\hline DMS (5\%) & & & 3, & & & 07 \\
\hline $\begin{array}{l}\pi=\text { dias apó } \\
\text { maiúscula na } \\
\text { pelo teste } t \text { a } 5 \\
=\text { não significa }\end{array}$ & P & dade. & $5^{12}$ & $\begin{array}{l}\text { dias } \\
\text { ferem } \\
\text { cative }\end{array}$ & $\overline{\mathrm{de}}$ & $\begin{array}{l}\text { ma letra } \\
\text { entre si } \\
<0,05 ; \text { NS }\end{array}$ \\
\hline
\end{tabular}


Aos 7 DAA não foram observadas diferenças substanciais no controle entre os tratamentos, o que ocorreu nas demais avaliações, em que, aos 14 DAA o Equip Plus + Atrazinax e o Sanson + Atrazinax foram de controle inferior ao Soberan (com e sem Atrazinax), tal qual ocorreu aos 50 DAA.

Aos 28 DAA o Equip Plus + Atrazinax foi de controle inferior aos demais tratamentos, e Sanson + Atrazinax e Callisto + Primóleo foram de resultados inferiores aos verificados aos tratamentos com Soberan. Para esse último produto não foram observadas diferenças entre as doses e vantagem da adição ou não de atrazina para o controle de $B$. plantaginea. Constantin et al. (2006) e Blanco et al. (2006) também avaliaram o controle do Soberan para B. plantaginea e observaram controle superior a $90 \%$ tanto para o Soberan isolado como em mistura com atrazina, o que mostra a efetividade desse herbicida no controle dessa espécie.

A espécie Digitaria horizontalis não foi satisfatoriamente controlada pelos herbicidas atrazine e oxyfluorfen em nenhuma das vias de absorção e tipos de solo (Tabela 2). Entretanto, para o oxyfluorfen em solo argiloso, houve uma significativa superioridade da via foliar em 3,1; 3,4 e 3,2 vezes em relação à radicular aos 3,7 e 14 DAA, respectivamente. Ao contrário da atrazine e do oxyfluorfen, o isoxaflutole proporcionou controle eficiente da $D$. horizontalis aos 14 DAA através das vias de absorção radicular e radicular + foliar (Tabela 2). A partir dos 7 DAA, a absorção radicular de isoxaflutole caracterizou por controle significativamente superior à via foliar, na ordem de 2,0 e 5,8 vezes, para os solos de textura arenosa e argilosa, respectivamente.
O controle de D. horizontalis aos 14 DAA, através da absorção radicular de isoxaflutole em solo de textura argilosa, apresentou-se altamente eficiente, superando significativamente a condição de solo arenoso, no qual o controle foi apenas satisfatório. Para a mesma espécie, apesar da absorção foliar de isoxaflutole não ter atingido controle satisfatório aos 14 DAA, na condição de solo arenoso, ao contrário da radicular, o nível de controle foi superior ao registrado para o solo de textura argilosa. Essa influência do tipo do solo na eficácia das diferentes formas de absorção de isoxaflutole para $D$. horizontalis não foi constatado para $B$. decumbens (Tabela 1 ), o que pode indicar a existência de uma sensibilidade diferencial entre espécies de plantas daninhas em relação ao modo de ação do herbicida. Entretanto, para os herbicidas atrazine e oxyfluorfen, apesar não de ter obtido eficiência satisfatória no controle de $B$. decumbens e $D$. horizontalis, mesmo sendo recomendados para ambas as espécies por Andrei (1999), Rodrigues \& Almeida (2005) e Lorenzi (2006) para as aplicações convencionais, a condição de solo argiloso indicou superioridade em relação ao solo arenoso para o controle de $B$. decumbens através da via radicular.

Os valores médios da matéria seca da parte aérea de $B$. decumbens (Tabela 3) e $D$. horizontalis (Tabela 4) foram coerentes com os dados de controle supramencionados, confirmando a intensidade dos efeitos provocados pelos diferentes herbicidas e vias de absorção. Ademais, verificou-se que quanto melhor a performance das vias de absorção, menor foi o acúmulo de matéria seca da parte aérea das plantas daninhas. 
Tabela 2. Controle (\%) de Digitaria horizontalis (DIGHO), considerando as diferentes possibilidades de absorção de atrazine, isoxaflutole e oxyfluorfen e as características dos solos.

\begin{tabular}{|c|c|c|c|c|c|c|}
\hline \multirow{3}{*}{$\begin{array}{l}\text { Formas de } \\
\text { Absorção }\end{array}$} & \multicolumn{6}{|c|}{ Controle $(\%)$ de DIGHO através da absorção de atrazine } \\
\hline & \multicolumn{2}{|c|}{$3 \mathrm{DAA}^{71^{-}}$} & \multicolumn{2}{|c|}{$7 \mathrm{DAA}$} & \multicolumn{2}{|c|}{$14 \mathrm{DAA}$} \\
\hline & Arenoso & Argiloso & Arenoso & Argiloso & Arenoso & Argiloso \\
\hline Radicular & $3,2 b^{12}$ & $2,4 \mathrm{~b}$ & $3,0 \mathrm{c} \mathrm{A}$ & $5,0 \mathrm{~b} \mathrm{~A}$ & $5,0 \mathrm{c}$ & $10,0 \mathrm{ab}$ \\
\hline Foliar & $4,2 \mathrm{ab}$ & $3,0 \mathrm{~b}$ & $7,0 \mathrm{~b} \mathrm{~A}$ & $5,0 \mathrm{~b} \mathrm{~A}$ & $9,0 \mathrm{~b}$ & $9,0 \quad b$ \\
\hline Radicular+foliar & $5,2 \mathrm{a}$ & $5,2 \mathrm{a}$ & 12,0 a $\mathrm{A}$ & 8,0 a B & $13,0 \mathrm{a}$ & $12,0 \mathrm{a}$ \\
\hline Testemunha & $0,0 \mathrm{c}$ & $0,0 \mathrm{c}$ & $0,0 \mathrm{~d} A$ & $0,0 \mathrm{c} \mathrm{A}$ & $0,0 \mathrm{~d}$ & $0,0 \quad \mathrm{c}$ \\
\hline$\overline{\text { Absorção (A) }}$ & \multicolumn{2}{|c|}{$49,91 *$} & \multicolumn{2}{|c|}{$172,75^{*}$} & \multicolumn{2}{|c|}{$100,09 *$} \\
\hline Solo $(\mathrm{S})$ & \multicolumn{2}{|c|}{$2,63^{\mathrm{NS}}$} & \multicolumn{2}{|c|}{$11,02 *$} & \multicolumn{2}{|c|}{$3,32^{\mathrm{NS}}$} \\
\hline $\mathrm{A} \times \mathrm{S}$ & \multicolumn{2}{|c|}{$0,94^{\mathrm{NS}}$} & \multicolumn{2}{|c|}{$5,03 *$} & \multicolumn{2}{|c|}{$6,93 *$} \\
\hline $\mathrm{CV} \%$ & \multicolumn{2}{|c|}{33,58} & \multicolumn{2}{|c|}{36,25} & \multicolumn{2}{|c|}{22,68} \\
\hline DMS $(5 \%)$ & \multicolumn{2}{|c|}{1,26} & \multicolumn{2}{|c|}{2,38} & \multicolumn{2}{|c|}{2,24} \\
\hline Formas de & \multicolumn{6}{|c|}{ Controle (\%) de DIGHO através da absorção de isoxaflutole } \\
\hline \multirow[t]{2}{*}{ Absorção } & \multicolumn{2}{|c|}{$3 \mathrm{DAA}^{7 \Gamma^{-}}$} & \multicolumn{2}{|c|}{$7 \mathrm{DAA}$} & \multicolumn{2}{|c|}{$14 \mathrm{DAA}$} \\
\hline & Arenoso & Argiloso & Arenoso & Argiloso & Arenoso & Argiloso \\
\hline Radicular & $17,6 \mathrm{~b} \mathrm{~A}$ & 19,8 a $\mathrm{A}$ & $54,4 \mathrm{~b}$ & $70,4 \mathrm{a}$ & $86,0 \mathrm{~b} \mathrm{~B}$ & 97,4 a $\mathrm{A}$ \\
\hline Foliar & $15,0 \mathrm{~b} \mathrm{~A}$ & $3,60 \mathrm{~b} \mathrm{~B}$ & $27,0 \mathrm{c}$ & $12,2 \mathrm{~b}$ & 44,8 c A & $17,4 \mathrm{~b} \mathrm{~B}$ \\
\hline Radicular+foliar & 26,8 a $\mathrm{A}$ & 20,8 a B & $68,6 \mathrm{a}$ & $76,6 \mathrm{a}$ & 98,8 a $\mathrm{A}$ & 99,6 a $\mathrm{A}$ \\
\hline Testemunha & $0,0 \mathrm{c} \mathrm{A}$ & $0,0 \mathrm{~b} \mathrm{~A}$ & $0,0 \mathrm{~d}$ & $0,0 \mathrm{c}$ & $0,0 \mathrm{~d} A$ & $0,0 \mathrm{c} \mathrm{A}$ \\
\hline Absorção (A) & \multicolumn{2}{|c|}{$60,32 *$} & \multicolumn{2}{|c|}{$195,29 *$} & \multicolumn{2}{|c|}{$501,85^{*}$} \\
\hline Solo $(\mathrm{S})$ & & & & & & \\
\hline$A \times S$ & 5 , & & & & & \\
\hline $\mathrm{CV} \%$ & & & & & & \\
\hline DMS $(5 \%)$ & & & & & & \\
\hline Formas & Contro & e $(\%)$ de $D$ & GHO atra & és da absc & ção de ox & luorfen \\
\hline Absorção & $3 \mathrm{D}$ & $\mathrm{A}^{7 \Gamma^{-}}$ & & & 14 & $\mathrm{AA}$ \\
\hline & $\overline{\mathrm{Arg}}$ & Oso & Arg & loso & Arg & oso \\
\hline Radicular & & & & & & \\
\hline Foliar & & & & & & \\
\hline Radicular+foliar & 22 & & & & & \\
\hline Testemunha & & & & & & \\
\hline Absorção (A) & 87 & & & & & \\
\hline $\mathrm{CV} \%$ & & & & & & \\
\hline DMS (5\%) & 3 , & & & & & \\
\hline $\begin{array}{l}\pi=\text { dias apó } \\
\text { maiúscula na } \\
\text { pelo teste } t \text { a } 5 \\
=\text { não significa }\end{array}$ & OrOo & $\begin{array}{l}\text { ula } \\
\text { ade }\end{array}$ & I & $\begin{array}{l}\text { lias se } \\
\text { ferem }\end{array}$ & $\mathrm{m}$ & $\begin{array}{l}\text { na letra } \\
\text { entre si } \\
0,05 ;\end{array}$ \\
\hline
\end{tabular}

De forma contrária, a característica matéria seca das raízes de $B$. decumbens
(Tabela 3) e D. horizontalis (Tabela 4) não apresentou a mesma viabilidade da matéria 
seca da parte aérea como ferramenta de análise subjetiva.

Tabela 3. Matéria seca da parte aérea e das raízes de Brachiaria decumbens (BRADC), considerando as diferentes possibilidades de absorção de atrazine, isoxaflutole e as características dos solos.

\begin{tabular}{|c|c|c|c|c|c|c|}
\hline \multirow{3}{*}{$\begin{array}{l}\text { Formas de } \\
\text { Absorção }\end{array}$} & \multicolumn{6}{|c|}{ Matéria Seca da parte aérea das plântulas de BRADC } \\
\hline & \multicolumn{2}{|c|}{ atrazine } & \multicolumn{2}{|c|}{ isoxaflutole } & \multicolumn{2}{|c|}{ oxyfluorfen } \\
\hline & Arenoso & Argiloso & Arenoso & Argiloso & Arenoso & Argiloso \\
\hline$\overline{\text { Radicular }}$ & $0,152 \mathrm{~b} \mathrm{~A}^{/ 1}$ & 0,133 c A & $0,104 \mathrm{~b} \mathrm{~A}$ & $0,109 \mathrm{~b} \mathrm{~A}$ & $0,129 \mathrm{~b} \mathrm{~A}$ & $0,121 \mathrm{~b} \mathrm{~A}$ \\
\hline Foliar & 0,133 b B & $0,273 \mathrm{~b} \mathrm{~A}$ & 0,103 b A & $0,104 \mathrm{~b} \mathrm{~A}$ & 0,102 c A & 0,087 с A \\
\hline Radicular+foliar & 0,033 с B & 0,124 c A & $0,101 \mathrm{~b} \mathrm{~A}$ & $0,127 \mathrm{~b} \mathrm{~A}$ & 0,094 c B & $0,136 \mathrm{~b} A$ \\
\hline Testemunha & 0,210 a B & 0,360 a A & 0,151 a B & 0,259 a A & $0,184 \mathrm{a} \mathrm{B}$ & 0,252 a $\mathrm{A}$ \\
\hline Absorção (A) & \multicolumn{2}{|c|}{$55,32^{*}$} & \multicolumn{2}{|c|}{$50,62 *$} & \multicolumn{2}{|c|}{$82,58^{*}$} \\
\hline Solo $(\mathrm{S})$ & \multicolumn{2}{|c|}{$58,38 *$} & \multicolumn{2}{|c|}{$26,48 *$} & \multicolumn{2}{|c|}{$12,90 *$} \\
\hline$A \times S$ & \multicolumn{2}{|c|}{$10,69 *$} & \multicolumn{2}{|c|}{$13,70 *$} & \multicolumn{2}{|c|}{$11,04 *$} \\
\hline $\mathrm{CV} \%$ & \multicolumn{2}{|c|}{21,45} & \multicolumn{2}{|c|}{16,32} & \multicolumn{2}{|c|}{13,72} \\
\hline DMS $(5 \%)$ & \multicolumn{2}{|c|}{0,177} & \multicolumn{2}{|c|}{0,028} & \multicolumn{2}{|c|}{0,024} \\
\hline Formas de & \multicolumn{6}{|c|}{ Matéria Seca das raízes das plântulas de BRADC } \\
\hline \multirow[t]{2}{*}{ Absorção } & \multicolumn{2}{|c|}{ atrazine } & \multicolumn{2}{|c|}{ isoxaflutole } & \multicolumn{2}{|c|}{ oxyfluorfen } \\
\hline & Arenoso & Argiloso & Arenoso & Argiloso & Arenoso & Argiloso \\
\hline Radicular & 2,144 a A & 2,174 a $\mathrm{A}$ & $0,463 \mathrm{a}$ & $0,391 \mathrm{~b}$ & $0,360 \mathrm{~b} \mathrm{~A}$ & $0,365 \mathrm{~b} \mathrm{~A}$ \\
\hline Foliar & 1,800 a B & 2,380 a A & $0,479 \mathrm{a}$ & $0,360 \mathrm{~b}$ & 0,257 b B & $0,415 \mathrm{~b} A$ \\
\hline Radicular+foliar & 1,072 b B & 1,886 a A & 0,469 a & $0,374 \mathrm{~b}$ & 0,290 b B & $0,451 \mathrm{~b} \mathrm{~A}$ \\
\hline Testemunha & 2,240 a A & 2,378 a A & $0,521 \mathrm{a}$ & $0,732 \mathrm{a}$ & 0,616 a B & $1,041 \mathrm{a} A$ \\
\hline Absorção (A) & \multicolumn{2}{|c|}{$7,57^{*}$} & \multicolumn{2}{|c|}{$7,34^{*}$} & \multicolumn{2}{|c|}{$45,25^{*}$} \\
\hline Solo $(\mathrm{S})$ & \multicolumn{2}{|c|}{$8,66^{*}$} & \multicolumn{2}{|c|}{$0,25^{\mathrm{NS}}$} & \multicolumn{2}{|c|}{$28,37 *$} \\
\hline$A \times S$ & \multicolumn{2}{|c|}{$1,93^{\mathrm{NS}}$} & \multicolumn{2}{|c|}{$4,21 *$} & & $7 *$ \\
\hline $\mathrm{CV} \%$ & 21 , & & 25 , & 11 & & 42 \\
\hline DMS (5\%) & 0,5 & & 0,1 & 54 & & 43 \\
\hline 11 & 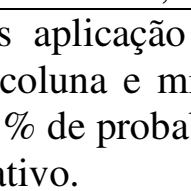 & idade & $\mathrm{s} .^{12}=$ & édias & $\mathrm{s}$ de & na letra \\
\hline
\end{tabular}

Desta forma, apenas a característica peso da matéria seca da parte aérea apresentou-se como informação complementar, garantindo assim maior confiabilidade nas interpretações dos dados de porcentagem de controle, em estudos das diferentes vias de absorção de herbicidas através desse método de avaliação.

Assim, conforme as condições de ausência de irrigação superficial após aplicação dos herbicidas e metodologia utilizadas nesse trabalho, os resultados sugerem que o isoxaflutole $\left(0,06 \mathrm{~kg}\right.$ i.a. ha $\left.{ }^{-1}\right)$ pode controlar de forma excelente a $B$. decumbens através da absorção foliar ou radicular, não havendo diferenças de eficácia entre os solos de textura arenosa e argilosa. Ao contrário, para $D$. horizontalis foi constatada a predominância da eficácia de controle para absorção radicular do isoxaflutole em relação à via foliar; tendo essa 
também sido caracterizada como excelente e textura argilosa, aos 14 DAA. significativamente superior para o solo de

Tabela 4. Matéria seca da parte aérea e das raízes de Digitaria horizontalis (DIGHO), considerando as diferentes possibilidades de absorção de atrazine, isoxaflutole e as características dos solos.

\begin{tabular}{|c|c|c|c|c|c|}
\hline \multirow{3}{*}{$\begin{array}{l}\text { Formas de } \\
\text { Absorção }\end{array}$} & \multicolumn{5}{|c|}{ Matéria Seca da parte aérea das plântulas de DIGHO } \\
\hline & \multicolumn{2}{|c|}{ atrazine } & \multicolumn{2}{|c|}{ isoxaflutole } & \multirow{2}{*}{$\begin{array}{c}\text { oxyfluorfen } \\
\text { Argiloso } \\
\end{array}$} \\
\hline & Arenoso & Argiloso & Arenoso & Argiloso & \\
\hline Radicular & 1,878 a $\mathrm{A}$ & 0,595 b B & 0,419 с A & 0,149 с B & $0,140 \mathrm{~b}$ \\
\hline Foliar & 2,256 a A & $0,868 \mathrm{ab} B$ & $1,086 \mathrm{~b} \mathrm{~A}$ & $0,651 \mathrm{~b} \mathrm{~B}$ & $0,170 \mathrm{a}$ \\
\hline Radicular+foliar & $0,970 \mathrm{~b} \mathrm{~A}$ & $0,561 \mathrm{~b} \mathrm{~A}$ & $0,166 \mathrm{~d} A$ & 0,144 с A & $0,172 \mathrm{a}$ \\
\hline Testemunha & 1,758 a $\mathrm{A}$ & $1,302 \mathrm{a} \quad \mathrm{A}$ & 2,036 a A & 1,412 a B & $0,184 \mathrm{a}$ \\
\hline Absorção (A) & \multicolumn{2}{|c|}{$7,71 *$} & \multicolumn{2}{|c|}{$261,90^{*}$} & $2,674^{*}$ \\
\hline Solo $(\mathrm{S})$ & \multicolumn{2}{|c|}{$44,27 *$} & \multicolumn{2}{|c|}{$58,44^{*}$} & - \\
\hline$A \times S$ & \multicolumn{2}{|c|}{$3,88 *$} & \multicolumn{2}{|c|}{$8,32 *$} & - \\
\hline $\mathrm{CV} \%$ & \multicolumn{2}{|c|}{32,98} & \multicolumn{2}{|c|}{17,96} & 9,22 \\
\hline DMS (5\%) & \multicolumn{2}{|c|}{0,54} & \multicolumn{2}{|c|}{0,18} & 0,02 \\
\hline Formas de & \multicolumn{5}{|c|}{ Matéria Seca das raízes das plântulas de DIGHO } \\
\hline \multirow[t]{2}{*}{ Absorção } & \multicolumn{2}{|c|}{ atrazine } & \multicolumn{2}{|c|}{ isoxaflutole } & oxyfluorfen \\
\hline & Arenoso & Argiloso & Arenoso & Argiloso & Argiloso \\
\hline Radicular & 7,009 b A & $2,177 \quad b$ B & $5,086 \mathrm{~b} \mathrm{~A}$ & $0,600 \mathrm{~b} \mathrm{~B}$ & 0,394 \\
\hline Foliar & 9,599 a $\mathrm{A}$ & $3,029 \mathrm{ab} \mathrm{B}$ & $5,375 \mathrm{~b} \mathrm{~A}$ & 1,882 b B & 0,378 \\
\hline Radicular+foliar & $6,055 \mathrm{~b} \mathrm{~A}$ & 1,353 b B & 1,298 c A & $0,522 \mathrm{~b} \mathrm{~A}$ & 0,288 \\
\hline Testemunha & 9,869 a A & $4,604 \mathrm{a} \quad \mathrm{B}$ & 10,078 a A & 4,386 a B & 0,358 \\
\hline$\overline{\text { Absorção (A) }}$ & \multicolumn{2}{|c|}{$8,367 *$} & \multicolumn{2}{|c|}{$39,61 *$} & $1,44^{\mathrm{NS}}$ \\
\hline Solo $(\mathrm{S})$ & \multicolumn{2}{|c|}{$92,80^{*}$} & \multicolumn{2}{|c|}{$73,43 *$} & - \\
\hline$A \times S$ & \multicolumn{2}{|c|}{$0,59^{\mathrm{NS}}$} & \multicolumn{2}{|c|}{$6,20 *$} & - \\
\hline $\mathrm{CV} \%$ & \multicolumn{2}{|c|}{32,10} & \multicolumn{2}{|c|}{36,37} & 21,99 \\
\hline DMS (5\%) & \multicolumn{2}{|c|}{2,27} & \multicolumn{2}{|c|}{1,72} & 0,12 \\
\hline$=$ dias & 2 & 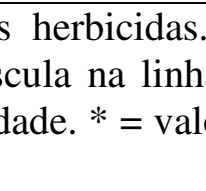 & $g n$ & $\cdot 1$ & $\begin{array}{l}\text { ma letra } \\
\text { entre si } \\
<0,05 ;\end{array}$ \\
\hline
\end{tabular}

Para o oxyfluorfen $\left(0,72 \mathrm{~kg}\right.$ i.a. ha $\left.{ }^{-1}\right)$, nas mesmas condições estudadas, apenas para B. decumbens caracterizou-se a superioridade da absorção foliar sobre a radicular (estruturas subterrâneas), não havendo distinção de eficácia para textura do solo utilizado. Com atrazine $\left(3,00 \mathrm{~kg}\right.$ i.a. $\mathrm{ha}^{-1}+$ óleo mineral Assist $\left.^{\circledR} 0,5 \% \mathrm{v} / \mathrm{v}\right)$, o baixo nível de controle obtido para $B$. decumbens reforçam a idéia da necessidade do auxílio do efeito físico da precipitação ou irrigação após aplicação para um melhor posicionamento do herbicida no perfil do solo e, consequentemente, para que ocorra o predomínio da absorção radicular.

\section{Conclusões}

Em função dos dados obtidos pode-se concluir que a absorção foliar e radicular de isoxaflutole, aos 15 DAA (dias após 
aplicação), controlou eficientemente a Brachiaria decumbens nos solos de textura arenosa e argilosa.

Para a espécie Digitaria horizontalis, o isoxaflutole aos 15 DAA proporcionou controle eficiente através da via radicular, respectivamente, na ordem de 1,9 e 5,6 vezes, superior a via foliar, para os solos de textura arenosa e argilosa.

A absorção foliar de oxyfluorfen foi eficiente no controle de $B$. decumbens, sendo 16,8 e 15,1 vezes superiores a via radicular, respectivamente, para os solos de textura arenosa e argilosa.

A espécie DIGHO não foi satisfatoriamente controlada pelos herbicidas atrazine e oxyfluorfen por nenhuma das diferentes vias de absorção e tipos de solo.

\section{Referências}

ADORYAN et al. Eficácia de isoxaflutole no controle de plantas daninhas na cultura da batata. Revista Brasileira de Herbicidas, v.3, n.2/3, p.133-138, 2002.

AL KHATIB, K; PARKER, R.; FUERST, E.P. Foliar absorption and translocation of herbicides from aqueous solution and treated soil. Weed Science, v.40, n.2, p.281-287, 1992.

ALMEIDA, F.S. Aplicações seqüenciais no controle de infestantes na cultura do milho. Londrina: IAPAR, 1989. p.10 (Informe da Pesquisa, 87).

ANDREI, E. Compêndio de defensivos agrícolas: guia prático de produtos fitossanitários para uso agrícola. 6 ed. São Paulo: Organização Andrei Editora Ltda., 1999. 671p.
DEUBER, R. Ciências das plantas infestantes: fundamentos. Jaboticabal: FUNEP, 2003. 452p.

DEVINE, M.D.; DUKE, S.O.; FEDTKE, C. Physiology of herbicide action. Englewood Cliffs: PRT Prentice Hall, 1993. 441p.

DEVINE, M.D. et al. Uptake and accumulation of the herbicides chlorsulfuron and clopyralid in excised pea root tissues. Plant Physiology, v.85, n.1, p.82-86, 1987.

GASKIN, R.E.; HOLLOWAY, P.J. Some physicochemical factors influencing foliar uptake enhancement of glyphosate-mono (isopropylammonium) by polyoxyethylene surfactants. Pesticide Science, v.34, n.3, p.195-206, 1992.

KARAM, D.; MELHORANÇA, A.L. A cultura do milho. Plantas daninhas. Sete Lagoas: Embrapa, 2004. Disponível em:<http://www.cnpms.embrapa.br/milho/ Importância.htm> Acesso em: 2 de fevereiro 2006.

KISSMANN, K.G. Plantas infestantes e nocivas. São Paulo: Basf, 1991. 608p.

LICHTNER, F.T. Amitrole absorption by bean (Phaseolus vulgaris L. cv. Red Kidney) roots. Mechanism of absorption. Plant Physiology, v.71, n.1, p.307-312, 1983.

LORENZI, H. Manual de identificação e controle de plantas daninhas: plantio direto e convencional. $6^{\text {a }}$ ed. Nova Odessa: Plantarum, 2006, p.190.

MACIEL, C.D.G. et al. Método alternativo de avaliação da absorção de atrazine por plantas de Brachiaria plantaginea. Planta Daninha, v.20, n.3, p.431-438, 2002. 
MARTINS, D. Interferência de capim marmelada na cultura da soja. Planta Daninha, v.12, n.1, p.144-151, 1994.

MEROTTO JR, A. et al. Aumento da população de plantas daninhas e uso de herbicidas no controle de plantas daninhas em milho. Planta Daninha, v.15, n.2, p.141-151, 1997.

PALLET, K.E. et al. The mode of action of isoxaflutole: 1. physiological effects, metabolism, and selectivity. Pesticide Biochemical Physiology, v. 62, n.2, p.113124, 1998.

PROCÓPIO, S.O. et al. Manejo de plantas daninhas na cultura da cana-de-açúcar. Viçosa: UFV, 2003, 150p.

RODRIGUES, B.N.; ALMEIDA, F.S. Guia de Herbicidas. 5 ed. Londrina, ed. dos autores, 2005. 648p.

SANTOS, C.A.L.; ROZANSKI, A. Controle de plantas daninhas na cultura do milho (Zea mays L.) por meio de herbicidas. Planta Daninha, v.2, n.2, p.120-123, 1979.

SILVA, A.A. et al. Controle de plantas daninhas. Brasília: ABEAS, 2000. 260p.

SOCIEDADE BRASILEIRA DA CIÊNCIA DAS PLANTAS DANINHAS. Procedimentos para instalação, avaliação e análise de experimentos com herbicidas. Londrina: SBCPD, 1995. 42p.

SOUZA, L.C.F. et al. Efeito da antecedência de gradagens ao plantio do milho no controle de plantas daninhas. Pesquisa Agropecuária Brasileira, v.31, n.11, p.789-793, 1996.

SPACER, V.; VIDAL, R.A. Eficácia de herbicidas graminícidas aplicados em pré- emergência no sistema de semeadura direta do milho. Planta Daninha, v.18, n.2, p.373-380, 2000.

SPRAGUE; C.L.; PENNER, D.; KELLS, J.J. Physiological basis for tolerance of four Zea mays hybrids to RPA 201772. Weed Science, v.47, n.4, p.375-382, 1999.

VIDAL, R.A. Herbicidas: mecanismo de ação e resistência de plantas. Porto Alegre: Palotti, 1997. 165p.

VIDAL, R.A.; MEROTTO JR, A. Herbicidologia. Porto Alegre: Evangraf, 2001, 152p.

WAHLERS, R.L. et al. Physiological characteristics of a stem cut and blade delivery method of application. Weed Science, v.45, n.6, p.746-749, 1997.

YOUNG, B.G.; HART, S.E. Mobility of RPA 201772 in Setaria faberi. Weed Science, v.48, n.2, p.188-192, 2000. 